\title{
PROJECTIVE BGG EQUATIONS, ALGEBRAIC SETS, AND COMPACTIFICATIONS OF EINSTEIN GEOMETRIES
}

\author{
A. ČAP, A.R. GOVER, M. HAMMERL
}

\begin{abstract}
For curved projective manifolds we introduce a notion of a normal tractor frame field, based around any point. This leads to canonical systems of (redundant) coordinates that generalise the usual homogeneous coordinates on projective space. These give preferred local maps to the model projective space that encode geometric contact with the model to a level that is optimal, in a suitable sense. In terms of the trivialisations arising from the special frames, normal solutions of classes of natural linear PDE (so-called first BGG equations) are shown to be necessarily polynomial in the generalised homogeneous coordinates; the polynomial system is the pull back of a polynomial system that solves the corresponding problem on the model. Thus questions concerning the zero locus of solutions, as well as related finer geometric and smooth data, are reduced to a study of the corresponding polynomial systems and algebraic sets. We show that a normal solution determines a canonical manifold stratification that reflects an orbit decomposition of the model. Applications include the construction of structures that are analogues of Poincaré-Einstein manifolds.
\end{abstract}

ARG gratefully acknowledges support from the Royal Society of New Zealand via Marsden Grant no. 06-UOA-029; AC and MH gratefully acknowledge support by project P19500-N13 of the "Fonds zur Förderung der wissenschaftlichen Forschung" (FWF) and the hospitality of the University of Auckland.

\section{INTRODUCTION}

Natural (or geometric) partial differential equations (PDE) are those which, in a suitable sense, are determined by some underlying geometry. Given such an equation, an important problem is to expose the geometric content of a solution, and the implications of its existence. Example questions close to our focus here are the following. What general results can be established concerning the nature and geometric structure of the solution's zero locus? What is the relationship of these features to the ambient structure? At the most primitive level there are questions of topology and smoothness; at the next level, it can be that the zero locus satisfies an interesting smooth embedding equation and inherits a rich intrinsic geometric structure.

Here we study a large class of solutions to overdetermined PDE arising naturally in projective geometry. We show that, to a surprising extent, these problems can be reduced to vastly simpler questions of an algebraic geometric type. This leads to a conceptual and practical way to describe, and manage geometrically, compactifications of curved geometries; the compactifications involved are naturally related to the geodesic structure.

2000 Mathematics Subject Classification. Primary 53B10, 53A20, 53C29, 35N10; Secondary 51N15, 53C30, 35Q75.

Key words and phrases. Projective differential geometry, compactifications, Poincaré-Einstein manifolds, Einstein manifolds, conformal geometry, parabolic geometries. 
Our work is partly inspired by the rich programme surrounding Poincaré-Einstein (PE) manifolds; these were introduced by Fefferman-Graham as a tool for constructing conformal invariants [21]. A PE structure consists of a conformal compactification of a geodesically complete Einstein-pseudo-Riemannian manifold, and broadly the programme involves naturally relating geometry and fields on a conformal boundary with Einstein-Riemannian geometry and field theory on the interior. Over the past decade this problem has been a domain of intense interest and deep progress [1, 10, 22, 23, 34, 38, 42], in part the work has been driven by strong links with the AdS/CFT correspondence of Maldacena [41, 36], see e.g. [32, 35, 46]. The original PE construction was preceded by a Kähler-Einstein-CR geometry analogue [19, 20] and recently there has been work to extend the picture to quaternionic-Kähler metrics and beyond [8, 9].

It was observed in [26] that a PE manifold is the same as a conformal manifold equipped with a solution of a certain conformally invariant PDE; this solution having the property that its (necessarily smooth and embedded) zero locus is precisely the topological boundary. That result led to an effective approach to certain key problems for these structures, extension to the notion of almost Einstein manifolds [27, 28], and also methods for geometrically constructing, and partly characterising, examples of PE manifolds [30]. In 28] it is seen that the almost Einstein class also naturally includes asymptotically locally Euclidean (ALE) structures that admit isolated point conformal compactification; in fact the nature of the compactification is shown to be an easy consequence of the compatibility of Ricci-flatness with the governing conformal PDE.

Here we show that considering a class of solutions to similar, but essentially different equations, leads to a natural extension of these ideas. In fact the scope is broader than this suggests, as the wider perspective draws in considerable new phenomena. In the simplest class of cases the linear equations studied take the form

$$
\left(\nabla_{\left(a_{1}\right.} \nabla_{a_{2}} \cdots \nabla_{\left.a_{k+1}\right)}+\text { lower order terms }\right) \sigma=0,
$$

where $\nabla$ is an affine connection, $\sigma$ is a function and the $(\cdots)$ indicates taking the symmetric part over the enclosed indices. (Here, and in many places throughout, we use Penrose's abstract index notation [44.) These equations have a symmetry known as projective invariance that plays an important role. We see in Section 3.1 that certain solutions for the cases $k=1$ and $k=2$ do indeed lead to structures that are analogous to PE manifolds. In particular in Section 3.3 the $k=2$ case yields a curved analogue of the hyperbolic ball that has been earlier described from quite a different perspective as a case of a "projectively compact metric" in [23]. This is a manifold with boundary. As in the case of a PE manifold, the boundary has a canonical conformal structure and the interior has a geodesically complete (Riemannian) negative Einstein structure. However in this case a projective compactification is involved, which emphasises the role of geodesics; this is strictly different from conformal compactification thus the structure is not $\mathrm{PE}$, see Proposition 3.3. There are analogues for all signatures and the Lorentzian case should be of interest to the general relativity community. These examples also show rather clearly that although the equations we consider are linear, the integrability conditions for these can involve very interesting non-linear conditions (such as the Einstein equations), on which we obtain a new perspective.

Let us now be specific about the full class of equations we treat. We restrict our attention to natural equations on a projective manifold of dimension at least 2 . Recall that this consists of a manifold $M$ equipped with an equivalence class $p$ of affine connections (we write $(M, p)$ ); the class is characterised by the fact that two connections 
$\nabla$ and $\widehat{\nabla}$ in $p$ have the same geodesics up to parametrisation. A model structure is $n$ dimensional projective space $\mathbb{R}^{n}$, but to avoid issues of orientability we prefer to work with its double cover, the projective sphere $\mathbb{S}^{n}$. We view this as a homogeneous space for $G=S L(n+1, \mathbb{R})$ and write $P$ for isotropy subgroup of a point; so $P$ is a maximal parabolic subgroup and we may identify $\mathbb{S}^{n}$ with $G / P$. To each irreducible $G$-representation $\mathbb{V}$ there is on $\mathbb{S}^{n}$ a canonical finite resolution by linear differential operators [18] which is related to the algebraic resolutions from [7, 40],

$$
0 \rightarrow \mathbb{V} \rightarrow \mathcal{B}^{0} \stackrel{D}{\rightarrow} \mathcal{B}^{1} \rightarrow \cdots \mathcal{B}^{n} \rightarrow 0 .
$$

Here the $\mathcal{B}^{i}$ are irreducible (weighted) tensor bundles. For the differential operators in the sequence there are canonical curved analogues, that is, generalisations that exist and are invariant on general projective manifolds $(M, p)$ [17]. In particular, this is true for each first $B G G$ operator $D$, and we use the same terminology (and notation) for each corresponding curved analogue.

These projectively invariant first BGG operators give the equations we study. For these we consider the special class of so-called normal solutions; see Section 2.3. On projective manifolds there is a canonical normal Cartan connection on a higher frame bundle. The equivalent induced linear connections are termed (normal) tractor connections [6, 11; the tractor and Cartan connections are reviewed briefly in Section 2.2. Beginning with a tractor bundle induced from an irreducible $G$-representation, each parallel tractor is equivalent to a normal solution of a first BGG equation. On $\mathbb{S}^{n}$ all solutions are normal, but a priori on curved structures it is a restriction.

By their definition, normal solutions are related to holonomy reductions of the Car$\tan /$ tractor connection and from this perspective certain local aspects have been investigated in [3, 4]. There one sees that, on the one hand, the available holonomy groups restrict the range of curved cases (although the treatment there classifies irreducible holonomy algebras, and so is not exhaustive), but on the other hand within the allowed groups interesting geometric structures arise including various pseudo-Riemannian Einstein (mentioned above) and contact adapted projective structures in the sense of [24].

The main focus of the current article is to show how to access geometric and topological information via a remarkable connection to the model $\mathbb{S}^{n}$, and then a new understanding of the nature of the first BGG solutions there. Recalling that Möbius' homogeneous coordinates are a fundamental tool for the calculus on projective space, in Section 2.5 we find local curved analogues, see Lemma 2.4. Based around a point, these are determined uniquely by a normal tractor frame that we also build. The whole construction is canonical up to a freedom parametrised by the parabolic subgroup $P$. The generalised homogeneous coordinates lead to a diffeomorphism between the curved projective manifold $\left(M^{n}, p\right)$ and the model $\mathbb{S}^{n}$ that, together with the normal frame, encodes a high degree of geometric contact. In particular, in the normal trivialisations, the components of parallel tractor fields on $(M, p)$ pull back to parallel tractor fields on the model, see Theorem 2.5 and Corollary 2.6 which are the first main results.

An immediate consequence of these constructions is Corollary 2.7 which shows that the components of normal solutions are necessarily the push forward (via the diffeomorphism) of a first BGG solution on the model. It follows that many local analytic and geometric questions for normal solutions on $(M, p)$ can be settled by studying the same problem in the simpler setting of the model. This strongly suggests there is significant value in understanding the nature of first BGG solutions on $\mathbb{S}^{n}$. It turns out that the answer, given in Proposition 2.9, is rather appealing: the first BGG solutions on $\mathbb{S}^{n}$ are precisely 
Čap, Gover, Hammerl

the (weighted) irreducible tensor fields arising from a natural class (that we term $G$ irreducible) of homogeneous polynomial systems on $\mathbb{R}^{n+1}$. For example, for a given $k$, the solutions of (1.1), on $\mathbb{S}^{n}$, are simply the projective polynomials of degree $k$. Remarkably in the curved setting, normal first BGG solutions are given by the same formal polynomial systems, now understood as polynomials in the generalised homogeneous coordinates. In general, these constructions allow us to conclude that a normal solution $\tau$ of a first BGG operator is, in a precise way, a curved analogue of a $G$-irreducible polynomial tensor field on $\mathbb{S}^{n}$. This is an interpretation of the next main result, Theorem 2.10 ,

To indicate the scope we point out that appropriate collections of the solutions of the BGG operators on scales (1.1) are sufficient to yield curved analogues of any projective polynomial system see Section 2.6. Note that since we work over $\mathbb{R}$, a polynomial system generally contains strictly more information that the algebraic set it determines. In particular various distinct geometric structures arise as curved analogues of distinct polynomial polynomial systems with the common feature of empty zero locus; these can be important and interesting, and in the analogous conformal setting this includes Fefferman spaces [13, 14, and positive Riemannian Einstein metrics [28].

As an immediate application, in Corollary 2.12 we see that the local nature of the zero locus of a normal solution $\tau$ may be completely deduced from the data of the corresponding algebraic set on $\mathbb{S}^{n}$. For example we can use this to descibe classes of cases where any zero locus of $\tau$ is necessarily a smooth embedded submanifold. In fact information that is both finer and has global content is available. For a projective manifold $(M, p)$ equipped with a normal solution $\tau$ we obtain a decomposition, or more accurately stratification, of $(M, p)$ which reflects the corresponding Bruhat-type orbit decomposition of the model $\mathbb{S}^{n}$; this is termed a $P$-type decomposition. This perspective should be useful in developing curved analogues of the vector valued Poisson transforms (cf. for example [43]). Information of a more analytic nature can also be deduced from the model, and indeed in two of the examples of Section 3.1 we use this idea to show that the open $P$-types are geodesically complete.

Finally we should point out that we have selected here, for development in some detail, just part of a very general picture. It is essentially clear that a direct analogue of our constructions is possible for conformal geometry; the importance of parallel tractors is more established in the conformal setting [2] and examples include the Fefferman space [13, 14, 39] (as well as PE and almost Einstein geometries). In fact via a different approach related results can be established for all Cartan geometries, and thus in particular for all parabolic geometries [15].

We thank Robin Graham for pointing out his construction with Fefferman of structures equivalent to the Klein-Einstein manifolds, which arise among the examples of Section 3.

\section{The CURVEd ANAlogue of PROJeCtive Polynomial Systems}

Here we shall construct and exploit curved analogues of certain projective polynomial systems. We require some background to describe the construction.

2.1. Projective differential geometry and tractor calculus. As mentioned above, we shall write $\mathbb{S}^{n}:=\mathbb{P}_{+}\left(\mathbb{R}^{n+1}\right)$ to denote the ray projectivisation of $\mathbb{R}^{n+1}$. This has a natural class of preferred paths that may be viewed as unparametrised geodesics; these arise from the projectivisation of 2-dimensional linear subspaces in $\mathbb{R}^{n+1}$. This structure is preserved by a group action. Evidently, $G:=S L(n+1, \mathbb{R})$ acts transitively on $\mathbb{S}^{n}$ and 
maps geodesics to geodesics. To be concrete in our development, we fix some choice of non-zero $e_{0} \in \mathbb{R}^{n+1}$ and define $P$ to be the parabolic subgroup stabilising the ray $\mathbb{R}_{+} \cdot e_{0}$.

The classical curved generalisation of $\mathbb{S}^{n}$ is termed a projective structure $\left(M^{n}, p\right)$, $n \geq 2$, as defined in the introduction. Alternatively phrased, as connections on $T^{*} M$, the elements in $p$ satisfy

$$
\widehat{\nabla}_{a} u_{b}=\nabla_{a} u_{b}-\Upsilon_{a} u_{b}-\Upsilon_{b} u_{a}
$$

where $\Upsilon$ is some smooth section of $T^{*} M$.

2.2. Projective tractor calculus. If $M$ is oriented we write $\mathcal{E}(1)$ for the $(-n-1)$ st root of the canonical bundle. Otherwise we write $\mathcal{E}(1)$ for a choice of line bundle with $(-2 n-2)$ nd power the square of the canonical bundle. We note that any connection $\nabla \in p$ determines a connection on $\mathcal{E}(1)$ and its real powers $\mathcal{E}(w), w \in \mathbb{R}$; we call $\mathcal{E}(w)$ the bundle of projective densities of weight $w$. As a point on notation: Given a bundle $\mathcal{B}$ we shall write $\mathcal{B}(w)$ as a shorthand for $\mathcal{B} \otimes \mathcal{E}(w)$.

Although, by the definition of a projective structure, there is no preferred connection on $T M$, there is a canonical connection, known as the tractor connection, on a related higher rank bundle. In the case of the model $\mathbb{S}^{n}=G / P$ this is remarkably simple. For any $P$-representation $\mathbb{W}$ one has the induced homogeneous bundle $\mathcal{W}:=G \times{ }_{P} \mathbb{W}$ where this means $G \times \mathbb{W}$ modulo the equivalence relation $(g r, v) \sim(g, r \cdot v)$, for $r \in P$. However in the special case that $\mathbb{W}$ is the restriction to $P$ of a $G$-representation then $\mathcal{W}$ is canonically trivialised $\phi: \mathcal{W} \rightarrow(G / P) \times \mathbb{W}$ by $(g, v) \mapsto(g P, g \cdot v)$. Canonically we have the trivial connection on $(G / P) \times \mathbb{W}$ and via $\phi$ this pulls back to the tractor connection $\nabla^{\mathcal{T}}$ on $\mathcal{W}$.

Since it occupies little space, and because in any case we need the notation and concepts, we review briefly the construction of the tractor connection in general; we follow [6] and the conventions there. In an abstract index notation let us write $\mathcal{E}_{A}$ for $J^{1} \mathcal{E}(1)$, the first jet prolongation of $\mathcal{E}(1)$. Canonically we have the jet exact sequence

$$
0 \rightarrow \mathcal{E}_{a}(1) \stackrel{Z_{A}^{a}}{\rightarrow} \mathcal{E}_{A} \stackrel{X^{A}}{\rightarrow} \mathcal{E}(1) \rightarrow 0
$$

where we have written $X^{A} \in \Gamma \mathcal{E}(1)$ for the jet projection, and $Z_{A}{ }^{a}$ for the map inserting $\mathcal{E}_{a}(1)$; these are both canonical. We write $\mathcal{E}_{A}=\mathcal{E}_{a}(1) \oplus \mathcal{E}(1)$ to summarise the composition structure in (2.2). As mentioned, any connection $\nabla \in p$ determines a connection on $\mathcal{E}(1)$, and this is precisely a splitting of (2.2). Thus given such a choice we have the direct sum decomposition $\mathcal{E}_{A} \stackrel{\nabla}{=} \mathcal{E}_{a}(1) \oplus \mathcal{E}(1)$ with respect to which we define a connection by

$$
\nabla_{a}^{\mathcal{T}}\left(\mu_{b} \mid \sigma\right):=\left(\nabla_{a} \mu_{b}+P_{a b} \sigma \mid \nabla_{a} \sigma-\mu_{a}\right) .
$$

Here $P_{a b}$ is the projective Schouten tensor and, with $R_{a b}{ }^{c} d$ denoting the curvature of $\nabla$, is related to the Ricci tensor $R_{a b}:=R_{c a}{ }^{c} b$ by $(n-1) P_{a b}=R_{a b}-\frac{2}{n+1} R_{[a b]} ;[\cdots]$ indicates the skew part over the enclosed indices. It turns out that (2.3) is independent of the choice $\nabla \in p$, and so $\nabla^{\mathcal{T}}$ is determined canonically by the projective structure $p$. This is the cotractor connection of [47] and is equivalent to the normal Cartan connection for the Cartan structure of type $(G, P)$, see [11. Thus we shall also term $\mathcal{E}_{A}$ the cotractor bundle, and we note the dual tractor bundle $\mathcal{E}^{A}$ (or in index free notation $\mathcal{T}$ ) has canonically the dual tractor connection: in terms of a splitting dual to that above this is given by

$$
\nabla_{a}^{\mathcal{T}}\left(\begin{array}{c}
\nu^{b} \\
\rho
\end{array}\right)=\left(\begin{array}{c}
\nabla_{a} \nu^{b}+\rho \delta_{a}^{b} \\
\nabla_{a} \rho-P_{a b} \nu^{b}
\end{array}\right)
$$


It will be useful to understand how the underlying connections in $p$ arise from the tractor connection. By dualising (2.2) it follows that the tractor bundle has a canonical composition structure given by the exact sequence

$$
0 \rightarrow \mathcal{E}(-1) \stackrel{X^{A}}{\rightarrow} \mathcal{E}^{A} \stackrel{Z_{\mathrm{A}}^{a}}{\rightarrow} \mathcal{E}^{a}(-1) \rightarrow 0 .
$$

The isomorphism $\mathcal{E}_{A} \stackrel{\nabla}{=} \mathcal{E}_{a}(1) \oplus \mathcal{E}(1)$ determined by $\nabla \in p$ also splits (2.5) and is evidently equivalent to a choice of section $Y_{B} \in \mathcal{E}_{B}(-1)$ satisfying $X^{B} Y_{B}=1$. Such a splitting is equivalent to a Weyl structure (cf. [16]). This determines a bundle monomorphism,

$$
Z^{A}{ }_{a}: \mathcal{E}^{a}(-1) \rightarrow \mathcal{E}^{A} \text {. }
$$

Using this a connection $\nabla_{a}^{Y}$ on $\mathcal{E}^{a}(-1)$ is then recovered from $\nabla^{\mathcal{T}}$ by the composition (on sections of $\left.\mathcal{E}^{a}(-1)\right)$ of $\nabla^{\mathcal{T}}$ with the map (2.6) followed by the canonical map $\mathcal{E}^{A} \rightarrow \mathcal{E}^{a}(-1)$ of (2.5); this is evident from (2.4). This then determines a connection on $T M$ that we may denote $\nabla^{Y}$.

Since $\mathcal{E}_{A}$ is $J^{1} \mathcal{E}(1)$ we have the canonical universal 1 -jet differential operator $\mathbb{D}_{A}$ : $\mathcal{E}(1) \rightarrow \mathcal{E}_{A}$, and from (2.2) $X^{A} \mathbb{D}_{A}$ is the identity on $\mathcal{E}(1)$. Thus any nonvanishing section $\sigma \in \Gamma \mathcal{E}(1)$ determines a special Weyl structure, termed a scale, by taking $Y_{A}:=\sigma^{-1} \mathbb{D}_{A} \sigma$. In fact by considering powers and roots of $\mathcal{E}(1)$ one sees that $D_{A}$ generalises to an invariant operator $\mathbb{D}_{A}: \mathcal{E}(w) \rightarrow \mathcal{E}_{A}(w-1), w \in \mathbb{R}$, known to Thomas [6, 47] and we shall term any non-vanishing section $\tau \in \Gamma \mathcal{E}(w), w \neq 0$, a scale (since we may take $\left.Y_{A}=\frac{1}{w} \tau^{-1} \mathbb{D}_{A} \tau\right)$. We write $\nabla^{\tau}$ for the affine connection in $p$ determined by a choice of scale $\tau$.

2.3. Normal solutions. Any vector bundle which is a tensor product of tensor powers of the tractor and cotractor bundles, or a tensor part thereof, is termed a tractor bundle. The structures which arise are handled efficiently by appeal to a principal bundle picture as follows.

Following [11] we consider the bundle $\mathcal{G}$ of adapted frames for $\mathcal{T}$ which respect the filtration structure shown in (2.5). This is a principal bundle with structure group $P$. Then tautologically $\mathcal{T}$ is the associated bundle $\mathcal{G} \times_{P} \mathbb{R}^{n+1}$. It is also straightforward to recover, from the tractor connection, the unique Cartan connection $\omega$ on $\mathcal{G}$ from which the tractor connection is induced. It follows that, given any representation $\mathbb{W}$ of $G$, we obtain a tractor bundle $\mathcal{W}=\mathcal{G} \times{ }_{P} \mathbb{W}$ equipped with a (linear) tractor connection induced from $\omega$. When we talk about tractor fields being parallel we mean that they are covariantly constant with respect to this connection. If $\mathbb{W}$ is an irreducible $G$-representation then we say that the tractor bundle $\mathcal{W}$ is $G$-irreducible. In this case there is a natural bundle map $\Pi: \mathcal{W} \rightarrow \mathcal{B}^{0}$, where $\mathcal{B}^{0}$ is an irreducible weighted tensor bundle, induced by the $P$-epimorphism from $\mathbb{W}$ to its $P$-irreducible quotient.

Proposition 2.1. [18] Let $\mathcal{V}$ be a $G$-irreducible tractor bundle on $(M, p)$ and suppose that $I$ is a parallel section of $\mathcal{V}$. Then the bundle map $\Pi: \mathcal{V} \rightarrow \mathcal{B}^{0}$ takes $I$ to a solution $\tau:=\Pi(I)$ of a first $B G G$ operator

$$
D: \mathcal{B}^{0} \rightarrow \mathcal{B}^{1}
$$

Definition: We shall say that $\tau$, arising as in the Proposition, is a normal solution (of the operator $D$ ). In the following text we may use the term "normal solution" to mean the normal solution for some first BGG operator $D$, without specifying $D$.

Remark: It is worth noting that in the case of the model $\mathbb{S}^{n}$ all solutions arise this way. In fact in the resolution (1.2) the $G$-representation $\mathbb{V}$ may be identified with the space of parallel tractors in the tractor bundle associated to $\mathbb{V}$. 
2.4. The Thomas cone space $\boldsymbol{M}$. In view of the canonical fibration $\underline{\pi}: \mathbb{R}^{n+1} \backslash 0 \rightarrow \mathbb{S}^{n}$ we may regard $\mathbb{R}^{n+1} \backslash 0$ as a cone space over $\mathbb{S}^{n}$. Here we recover the curved analogue of this (which was probably known to T.Y. Thomas), see [16, 24].

In Section 2.3 above we mentioned that the Cartan connection induces a canonical tractor connection on any associated bundle $\mathcal{G} \times{ }_{P} \mathbb{W}$, where $\mathbb{W}$ is the restriction to $P$ of a $G$-representation. It is immediate from the equivariance properties of $\omega$ that, more generally for any closed subgroup $P_{0} \subset P$, we obtain a canonical connection on $\mathcal{G} \times{ }_{P_{0}} \mathbb{W}$, a vector bundle over the fibrewise quotient $\mathcal{G} / P_{0}$. In particular, let us henceforth write $P_{0}$ to denote the subgroup of $G$ fixing $e_{0}$ and define $\boldsymbol{M}$ to be the quotient $\mathcal{G} / P_{0}$, that is, it is the total space

$$
M=\mathcal{G} \times_{P} \mathbb{E}_{+}
$$

where $\mathbb{E}_{+}$is the $\mathbb{R}_{+}$-ray generated by $e_{0}$ in $\mathbb{R}^{n+1}$; from (2.5) we see that it is equivalently the total space of the ray-bundle $\mathcal{E}(-1)_{+}$(i.e. the subbundle of positive rays in $\mathcal{E}(-1)$ ). We write $\pi: \boldsymbol{M} \rightarrow M$ for the canonical bundle projection.

Now observe that, as $P_{0}$ representations, we have $\mathfrak{g} / \mathfrak{p}_{0} \cong \mathbb{R}^{n+1}$. From this there follow two points. First by the last isomorphism, and that $\mathbb{R}^{n+1}$ may be considered as the restriction to $P_{0}$ of a $G$-representation space, it follows that $\omega$ canonically induces a vector bundle connection on $\mathcal{G} \times{ }_{P_{0}} \mathfrak{g} / \mathfrak{p}_{0}$. Second, by the standard theory of Cartan connections, we also have canonically the identification $\mathcal{G} \times P_{0} \mathfrak{g} / \mathfrak{p}_{0} \cong T \boldsymbol{M}$. From the formula for the tractor connection (equivalently the normalisation conditions of the normal Cartan connection) it follows that this connection is Ricci-flat. In summary.

Proposition 2.2. The projective structure $(M, p)$ determines a canonical Ricci-flat affine connection $\boldsymbol{\nabla}$ on the manifold $\boldsymbol{M}$.

The canonical section $X^{A}$ corresponds to a section $\zeta^{A}$ of $T \boldsymbol{M}$ which generates the $\mathbb{R}_{+}$ action on the fibres, and it is straightforward to verify that

$$
\nabla_{B} \zeta^{A}=\delta_{B}^{A}
$$

Associated bundles on $M$ arise from $P$-representations $\mathbb{U}$ as $\mathcal{G} \times{ }_{P} \mathbb{U}$. Sections are functions $u: \mathcal{G} \rightarrow \mathbb{U}$ which are $P$-equivariant in the sense that $u(g \cdot r)=r^{-1} \cdot u(g)$. Since $P$ equivariance trivially implies equivariance for any subgroup, it follows that, by restriction, such sections lift immediately to sections of the corresponding bundle $\mathcal{G} \times{ }_{P_{0}} \mathbb{U}$ over $\boldsymbol{M}$. In particular using the formula for the tractor connection from [11, Section 2.5] one sees immediately that, in the case that $\mathbb{U}$ is a $G$-representation, parallel tractor fields on $M$ correspond in an obvious way with parallel tensor fields on $\boldsymbol{M}$. It follows that arbitrary smooth sections of $\mathcal{T}$ (or $\mathcal{T}^{*}$ ) correspond to sections of $T \boldsymbol{M}$ (resp. $T^{*} \boldsymbol{M}$ ) that are in the null space of $\zeta^{A} \boldsymbol{\nabla}_{A}$, and so general (unweighted) tractor fields correspond in an obvious way to tensor fields that are parallel in the directions of the fibres of $\pi$. Using (2.7) and that $\boldsymbol{\nabla}$ is torsion free, this means that a section of $\mathcal{T}$ corresponds to a section of $T M$ which is homogeneous of degree -1 , with respect to the principal $\mathbb{R}_{+}$-action.

Now any section of $\pi$ determines a splitting of (2.5) and so a connection from $p$. Note that a scale $\sigma$ determines a unique section of $\mathcal{E}(-1)_{+}$and thus a section of $\pi$. It is straightforward to verify [16] that the affine connection $\nabla^{\sigma}$ that arises is related to the Thomas space connection $\boldsymbol{\nabla}$ as follows.

Lemma 2.3. Let $u, v \in \Gamma T M$ and $\sigma$ a scale viewed, as a section of $\pi: M \rightarrow M$. Then

$$
\nabla_{u} v=\pi_{*}\left(\nabla_{\sigma_{*} u} \sigma_{*} v\right)
$$


Note that (2.7) implies that $\zeta^{A} \nabla_{A} \zeta^{B}=\zeta^{B}$ so each fibre of $\pi$ agrees with the trace of a vertical geodesic. It follows that other geodesics remain transverse to the fibres for all time, and project to regular curves on $M$. It is an easy consequence of (2.3) that these are geodesics from the class on $(M, p)$.

2.5. Generalised homogeneous coordinates. Here we shall show that given a point $q \in M$, and a choice of adapted frame for $\mathcal{T}(1)_{q}$, we obtain an otherwise canonical diffeomorphism between $(\boldsymbol{M}, \boldsymbol{\nabla})$ and affine $\mathbb{R}^{n+1}$; this map is distinguished by its properies of geometric contact with the model, as we shall see later in this section.

Recall we denote by $\zeta$ the fundamental vector field generating the principal right $\mathbb{R}_{+}-$ action on $\boldsymbol{M}$. In the case of the model $\underline{\pi}: \mathbb{R}^{n+1} \backslash 0 \rightarrow \mathbb{S}^{n}$ the fundamental field coincides with the usual Euler vector field $E$, and the affine connection $\boldsymbol{\nabla}$ agrees with the usual affine parallel transport.

Lemma 2.4. Choose $\tilde{q} \in \boldsymbol{M}$, and a unit volume frame $e_{0}, \ldots, e_{n}$ for $T_{\tilde{q}} \boldsymbol{M}$, with $e_{0}=\zeta$. This determines a diffeomorphism $\Phi: \underline{\pi}^{-1}\left(U^{\prime}\right) \rightarrow \pi^{-1}(U)$ for some open neighbourhood $U$ of $q:=\pi(\tilde{q})$ and some open set $U^{\prime}$ in $\mathbb{S}^{n}$. With the following properties:

- $\Phi$ is $\mathbb{R}_{+}$-equivariant and so determines a diffeomorphism $\phi: U^{\prime} \rightarrow U$;

- $\Phi$ maps straight lines through $\Phi^{-1}(\tilde{q})$ to geodesics for $\boldsymbol{\nabla}$ through $\tilde{q}$, and so $\phi$ maps great circles through $\phi^{-1}(q)$ to geodesic paths through $q$;

- $\Phi^{*} \zeta$ is the Euler vector field on $\underline{\pi}^{-1}\left(U^{\prime}\right) \subset \mathbb{R}^{n+1}$.

Proof: We shall write exp for the affine exponential map of $\boldsymbol{\nabla}$ at the point $\tilde{q}$. Now let $W$ be an open neighborhood of zero in $\mathbb{R}^{n}$ such that

$$
\left(x^{1}, \ldots, x^{n}\right) \mapsto \pi\left(\exp \left(x^{1} e_{1}+\cdots+x^{n} e_{n}\right)\right)
$$

defines a diffeomorphism from $W$ onto an open neightborhood $U$ of $q:=\pi(\tilde{q})$ in $M$. We may identify $W$ with the affine hyperplane neighbourhood $\{(1, x): x \in W\}$ in $\mathbb{R}^{n+1}$, and write $U^{\prime} \subset \mathbb{S}^{n}$ for the open subset consisting of its image under $\underline{\pi}$. Now define a map $\Phi: \underline{\pi}^{-1}\left(U^{\prime}\right) \rightarrow \pi^{-1}(U)$ by

$$
\left(r, r x^{1}, \ldots, r x^{n}\right) \mapsto \exp \left(x^{1} e_{1}+\cdots+x^{n} e_{n}\right) \cdot r,
$$

where $r>0,\left(x^{1}, \ldots, x^{n}\right) \in W$ and the dot indicates the principal right action. Evidently, this is an $\mathbb{R}_{+}$-equivariant diffeomorphism, so it induces a diffeomorphism $\phi: U^{\prime} \rightarrow U$ and $\Phi^{*} \zeta$ is the Euler vector field on $\underline{\pi}^{-1}\left(U^{\prime}\right) \subset \mathbb{R}^{n+1}$. Also, $\phi$ maps great circles through $\underline{\pi}((1,0))$ to geodesic paths through $q$, since by construction it maps straight lines in the affine hyperplane through $(1,0)$ to geodesics through $\tilde{q}$ in $\boldsymbol{M}$.

Remark: Note that the frame $\left\{e_{0}, \ldots, e_{n}\right\}$ for $T_{\tilde{q}} \boldsymbol{M}$ determines an adapted frame for $\mathcal{T}_{q}$. Varying $\tilde{q} \in \pi^{-1}(q)$, any adapted frame can be obtained in this way. Hence at a given point $q \in M$, the freedom of choice is parametrised by $P$.

The Lemma leads to the following observation:

Remark: Generalised homogeneous coordinates. Let us write $X^{0}, X^{1}, \cdots, X^{n}: \pi^{-1}(U) \rightarrow$ $\mathbb{R}$ for the functions on $\pi^{-1}(U) \subset M$ which are the push forward via $\Phi$ (i.e. pull back via $\Phi^{-1}$ ) of the standard coordinates $X^{0}, X^{1}, \cdots, X^{n}$ on $\mathbb{R}^{n+1}$ (restricted to $(\underline{\pi})^{-1}\left(U^{\prime}\right)$ ). Since $\Phi$ is a diffeomorphism, the $X^{\underline{A}}, \underline{A}=0,1, \cdots, n$, are coordinates on $\pi^{-1}(U)$. Also note that by the equivariancy of $\Phi$, these functions are homogeneous of degree one for the principal $\mathbb{R}_{+}$-action on $\boldsymbol{M}$, so they are equivalent to 1 -densities on $M$. This collection of densities may be viewed as curved versions of homogeneous coordinates. 
2.6. The fundamental theorem for parallel tractors. We show here that the diffeomorphism of Lemma 2.4 captures a high degree of contact between $(M, p)$ and $\mathbb{S}^{n}$. This is observed by a compatibility between the tractor parallel transport, on the two manifolds, that we shall describe precisely. First we construct a frame field for $T \boldsymbol{M}$ on $\pi^{-1}(U)$ that corresponds to an adapted frame field for $\mathcal{T}$ on $U$.

Here we continue the notation of Lemma 2.4. Take the vectors $e_{1}, \ldots, e_{n}$ at $\tilde{q}$, and transport them parallely along the horizontal geodesics $t \mapsto \exp (t x)$ for $x$ in the span of $e_{1}, \ldots, e_{n}$. Possibly shrinking $U$, these vectors project onto a local frame $\left\{\xi_{1}, \ldots, \xi_{n}\right\}$ for the tangent bundle $T M$ over $U$.

Next, we claim that putting $e_{0}=\zeta$ along these horizontal geodescis, we obtain a unit volume frame $\left\{e_{0}, \ldots, e_{n}\right\}$ along $\exp (W)$. Let $c(t)$ be one of the horizontal geodesics through $\tilde{q}$. Then $c^{\prime}(t)$ is obtained by parallely transporting $c^{\prime}(0)$ along the geodesic to $c(t)$. By assumption, $c^{\prime}(0)$ lies in the span of $e_{1}, \ldots, e_{n}$, whence $c^{\prime}(t)$ lies in the span of $e_{1}(c(t)), \ldots, e_{n}(c(t))$. But together with $\nabla_{\xi} \zeta=\xi$ and $\nabla_{c^{\prime}(t)} e_{i}=0$ along $c(t)$, this implies that $\nabla_{c^{\prime}(t)}\left(\zeta \wedge e_{1} \wedge \cdots \wedge e_{n}\right)=0$ along $c(t)$. Since $\nabla$ is volume preserving, and the frame has unit volume in $\tilde{q}$, the claim follows.

Finally we extend our frame along the filbres of $\pi$ by requiring homogeneity of degree -1 with respect to the principal $\mathbb{R}_{+}$-action, that is we require $e_{i}(y \cdot r)=r^{-1} \rho_{*}^{r} \cdot e_{i}(y)$, where $\rho$ denotes the $\mathbb{R}_{+}$-action. Then it is clear by construction that $e_{i}$ defines a frame for the tangent bundle $T \boldsymbol{M}$ over $\pi^{-1}(U)$, and at the same time determines an adapted frame for $\mathcal{T}$ over $U$ via the correspondence of Section 2.4. Notice that by construction and the equivariancy of $\Phi$ we see that since $\Phi^{*} e_{0}$ equals the Euler vector field $E$ on $\{(1, x): x \in W\}$, we have $\Phi^{*} e_{0}=\left(X^{0}\right)^{-1} E$ on $\underline{\pi}^{-1}\left(U^{\prime}\right)$.

Next we need to known what this construction yields on the model $\mathbb{S}^{n}$. The natural choice is to take $\tilde{q}=(1,0) \in \mathbb{R}^{n+1}$ and $e_{\underline{A}}=\partial_{\underline{A}}=\frac{\partial}{\partial X \underline{A}}(\tilde{q})$ for $\underline{A}=1, \ldots, n$. It is easily concluded that the construction just gives the frame field $\left\{\frac{1}{X^{0}} E, \partial_{1}, \ldots, \partial_{n}\right\}$ on the half space $X^{0}>0$. Now a constant tensor $\boldsymbol{I}^{\prime}$ on $\mathbb{R}^{n+1}$ is equivalent to a parallel tractor on $I^{\prime}$ on $\mathbb{S}^{n}$; this uses Section 2.4 for the model. Putting these things togther we come to the following key fact. Here to simplify the statement, density bundles are trivialised by the scale $X^{0}$ (corresponding to working on the section $X^{0}=1$ of $\boldsymbol{M}$ ).

Theorem 2.5. Suppose that I is a parallel section of a tractor bundle. Composing with $\phi$ the coordinate functions of I with respect to the frame derived from $\left\{e_{0}, \ldots, e_{n}\right\}$, one obtains the coordinate functions of a parallel tractor $I^{\prime}$ on the homogenous model with respect to the tractor frame obtained in the same way from $\left\{\frac{1}{X^{0}} E, \partial_{1}, \ldots, \partial_{n}\right\}$.

Proof: Denote by $\boldsymbol{I}$ the parallel tensor on $(\boldsymbol{M}, \boldsymbol{\nabla})$ equivalent to $I$. We consider an expression for $\boldsymbol{I}$ in the form $a_{J} e_{J}$, where the elements $e_{J}$ are linear combinations of tensor products of the $e_{j}$, which form a local frame for the given tensor bundle. Then along any of the geodesics $c(t)$ through $\tilde{q}$, and lying in $\exp (W)$, we can consider

$$
0=\nabla_{c^{\prime}(t)} \sum_{J} a_{J} e_{J}=\sum_{J}\left(c^{\prime}(t) \cdot a_{J}(c(t))\right) e_{J}+\sum_{J} a_{J} \nabla_{c^{\prime}(t)} e_{J}
$$

To expand the last term, we only need to know that $\nabla_{c^{\prime}(t)} e_{0}=c^{\prime}(t)$ while $\nabla_{c^{\prime}(t)} e_{i}=0$ for $i>0$ along $c(t)$. This shows that for the coefficients $a_{J}$ we obtain a first order ODE on the function $t \mapsto a_{J}(c(t))=b_{J}(t)$ which has the form $b^{\prime}(t)=F(b(t))$ and one obtains the same system on the corresponding straight line through $(1,0) \in \mathbb{R}^{n+1}$ over the homogenous model $\mathbb{S}^{n}$. In vertical directions, everything is fixed by homogeneity, so we obtain the result. 
There is a useful variant of the above result. To simplify the discussion let us simply identify tractor fields in $M$ with the corresponding homogeneous tensor fields on $\boldsymbol{M}$ and do the same on the model.

On the homogeneous model, we can directly compute the change from the frame $\left\{\frac{1}{X^{0}} E, \partial_{1}, \ldots, \partial_{n}\right\}$ to $\left\{\partial_{0}, \ldots, \partial_{n}\right\}$, and then make the same change on $M$. Denoting, as above, the generalised homogeneous coordinates on $M$ by $X^{\underline{A}}$, this implies that any parallel tractor has constant coordinate functions with respect to the the frame

$$
\left\{f_{0}, f_{1}, \ldots, f_{n}\right\}, \quad \text { where } \quad f_{0}=e_{0}-\frac{X^{1}}{X^{0}} e_{1}-\cdots-\frac{X^{n}}{X^{0}} e_{n}
$$

and $f_{i}=e_{i}$ for $i=1, \cdots, n$. The corresponding coframe is given by $f^{0}=e^{0}$ and $f^{i}=e^{i}+\frac{X^{i}}{X^{0}} e^{0}$ for $i=1, \cdots, n$.

Since the change of frame (2.8) is rational in the coordinates and these coordinates are the push forward by $\Phi$ of the standard coordinates on $\mathbb{R}^{n+1}$, Theorem 2.5 can be equivalently phrased in terms of the frame $f_{\underline{A}}$. More generally we may define, on a sufficiently small neighbourhood $U$ of any point $q \in M$, a map $\bar{\Phi}$ from tractor fields on $U^{\prime}=\phi^{-1}(U) \subset \mathbb{S}^{n}$ to tractor fields on $U$ as follows: Use the frame $f_{\underline{A}}$ and its dual to trivialise the tensor bundles on $\boldsymbol{M}$. Use the standard $\mathbb{R}^{n+1}$ frame to do the same on the model. Then push forward the component functions of tractors fields (as homogeneous functions on $\mathbb{R}^{n+1}$ ) on the model via the diffeomorphism $\Phi$ and interpret as components of a tractor field in the trivialisation on $M$. Then we have the following.

Corollary 2.6. Given $q \in M$, a choice of adapted frame $e_{A}(q)$ for $\mathcal{T}_{q}$ canonically determines, for some neighbourhood $U$ of $q$, a diffeomorphism $\bar{\phi}: U^{\prime} \subset \mathbb{S}^{n} \rightarrow U$, a trivialisation of tractor and density bundles, and a compatible map $\bar{\Phi}$ from tractor fields on $U^{\prime}$ to tractor fields on $U$ with the properties:

- for parallel tractors I the component functions are constant;

- any parallel tractor $I$ on $U$ is the image under $\bar{\Phi}$ of a parallel tractor $U^{\prime} \subset \mathbb{S}^{n}$;

- the components of the canonical tractor field $X^{A}$ (in the trivialisation) are exactly the generalised homogeneous coordinates $X^{\underline{A}}$ of Section [2.5, and these are the image under $\bar{\Phi}$ of the standard coordinates on $\mathbb{R}^{n+1}$.

Proof: The first part of the last fact follows from (2.8) and that $X^{0} e_{0}=\zeta$. The final observation is immediate since on homogeneous functions on $\mathbb{R}^{n+1}, \bar{\Phi}$ is just $\Phi_{*}$. The other points were treated above.

Remark: By the same argument that led to the first bullet point of the Corollary, we see that the frame field $f_{A}$ is parallel along those geodesics through $\tilde{q} \in \boldsymbol{M}$ which lie in $\exp (W)$. Then in the vertical directions we have $\boldsymbol{\nabla}_{\zeta} f_{A}=0, \underline{A}=0,1, \cdots, n$. These properties with $f_{\underline{A}}(\tilde{q}):=e_{\underline{A}}(q)$ obviously characterise this frame, which we will call a normal frame. Thus the generalised homogeneous coordinates are characterised as the component functions (densities) of the canonical tractor $X^{A}$, with respect to this normal frame. This frame also determines a normal scale $\sigma=f_{A}^{0} X^{A}=e_{A}^{0} X^{A}$. This agrees with $\bar{\Phi}\left(X^{0}\right)$, so trivialising density bundles on $M$ using $\sigma$, is compatible via $\phi$ with trivializing density bundles on $\mathbb{S}^{n}$ using $X^{0}$. This is implicit in the construction proving Theorem 2.5 .

In our discussion below, we shall use both the normal frame $f_{A}$ and the adapted frame $e_{\underline{A}}$. Note that the map $\bar{\Phi}$ can also been obtained using the trivialisations corresponding to the adapted frame. 
Theorem 2.5 and its equivalent Corollary 2.6 allow us to treat normal solutions since, by Proposition 2.1. each such arises as $\Pi(I)$ for a parallel tractor $I$, where $\Pi$ denotes the projection $\Pi$ to the irreducible quotient bundle. This is easily understood using the adapted tractor frame $\left\{e_{0}, \cdots, e_{n}\right\}$ from above. Referring to (2.2) and (2.5), but using the normal scale $\sigma=e_{A}^{0} X^{A}$ to trivialise densities, the projection $Z_{A}{ }^{a}: \mathcal{E}^{A} \rightarrow \mathcal{E}^{a}$ to the irreducible quotient is characterised by $e_{0} \mapsto 0$ and $e_{i} \mapsto \xi_{i}$ for $i=1, \ldots, n$. Dually for $\mathcal{E}_{A}$ the projection is given by contracting with $X^{A}$, and so is characterised by by $e^{0} \mapsto 1$ and $e^{i} \mapsto 0$ for $i=1, \ldots, n$. These determine $\Pi$ on tensor products.

Let us describe the trivialisation of tensor bundles on $M$ (or $\mathbb{S}^{n}$ ) induced by the frame $\left\{\xi_{1}, \ldots, \xi_{n}\right\}$ and the normal density $\sigma$ (the frame on $\mathbb{S}^{n}$ given by the projection of $\left\{\partial_{1}, \ldots, \partial_{n}\right\}$ and the normal density $\left.X^{0}\right)$ as a normal trivialisation.

Corollary 2.7. Suppose that $\tau$ is a normal solution of a first $B G G$ equation $D \tau=0$ on $\left(M^{n}, p\right)$. For each point $q \in M$ there are open sets $U \ni q$ and $U^{\prime} \subset \mathbb{S}^{n}$ and a diffeomophism $\phi: U^{\prime} \rightarrow U$, which maps great circles through $\phi^{-1}(q)$ to geodesics through $q$, such that $\underline{\tau} \circ \phi=\underline{\tau}^{\prime}$ where $\tau^{\prime}$ is a solution for $D$ on the model, and $\underline{\tau}$, $\underline{\tau}^{\prime}$ are the component functions of $\tau$ and $\tau^{\prime}$ in the appropriate normal trivialisations.

The Corollary here is a first version of what we shall shortly refer to as the fundamental theorem for normal solutions. What is lacking at this point is some explicit understanding of what the normal BGG solutions are on the model. Fortunately the tools we have already developed give an answer almost immediately. Let us follow through a the above for a special class of examples.

Consider the case of a completely symmetric parallel tractor $H_{A_{1} \cdots A_{k}}$ on $(M, p)$. From (2.2) the map $\Pi$ of Proposition 2.1 is simply $H_{A_{1} \cdots A_{k}} \mapsto H_{A_{1} \cdots A_{k}} X^{A_{1}} \cdots X^{A_{k}}=: \sigma \in$ $\Gamma \mathcal{E}(k)$. Now $\sigma$ corresponds to degree $k$ homogeneous function on $\boldsymbol{M}$ that we shall denote the same way. From Corollary 2.7, locally (and using the constructions and notation from above) we have $\sigma=\phi_{*} \sigma^{\prime}$ where $\sigma^{\prime}$ is a solution to $D$ on $\mathbb{S}^{n}$. With respect to the trivialistions arising from the tractor frame $f_{\underline{A}}$, we have $\sigma$ corresponds to the homogeneous function $\sigma=H_{\underline{A}_{1} \cdots \underline{A}_{k}} X^{\underline{A}_{1}} \cdots X^{\underline{A}} \underline{A}_{k}$ on $\boldsymbol{M}$. From Corollary 2.6, the components $H_{\underline{A}_{1} \cdots \underline{A}_{k}}$ are constant, so $\sigma$ is expressed as a polynomial. According to the construction, and using Corollary 2.5, $\sigma^{\prime}$ is simply the same homogeneous polynomial (in standard coordinates), now viewed as a homogeneous function on $\mathbb{R}^{n+1}$. The latter is precisely a projective polynomial on $\mathbb{S}^{n}$ of degree $k$.

Note that this outcome is also clear via another perspective from Corollary 2.6. $\sigma=$ $\Pi(H)$ is $\bar{\Phi} \sigma^{\prime}$, for some density $\sigma^{\prime}$ on $\mathbb{S}^{n}$. By expressing $\sigma^{\prime}$ in the frame $f_{\underline{A}}$ as above, $\sigma=H_{\underline{A}_{1} \cdots \underline{A}_{k}} X^{\underline{A}} \underline{A}_{1} \cdots X^{\underline{A}} \underline{A}_{k}$, and using Corollary [2.6, we see $\sigma^{\prime}$ is given by the same formal expression on $\mathbb{R}^{n+1}$ and hence is $\Pi\left(H^{\prime}\right)$ for a parallel tractor $H^{\prime}$ there. From either persepective, as a special case of this we may replace $(M, p)$ with $\mathbb{S}^{n}$ to obtain the following.

Proposition 2.8. On $\mathbb{S}^{n}$ the degree $k$ projective polynomials are precisely the first $B G G$ solutions corresponding to symmetric rank $k$ parallel tractors.

So we see that, at least for this class of cases, first BGG solutions on the model are just projective polynomials. On the other hand Corollary 2.7 shows that corresponding normal solutions in $\Gamma \mathcal{E}(k)$, on a projective manifold $(M, p)$, are curved analogues. Since any algebraic set arises from a collection of such polynomials, we have a universal way to describe a canonical curved analogue of the projective polynomial system involved.

We may elaborate on the Proposition somewhat. The PDE involved are the equations of the operators $D_{k}: \Gamma \mathcal{E}(k) \rightarrow \Gamma \mathcal{E}_{\left(a_{1} a_{2} \cdots a_{k}\right)}(k)$ that, in terms of $\nabla \in p$, take the form 
(1.1). The equation $D_{k} \sigma=0$ characterises degree $k$ projective polynomial densities. The specific polynomial densities solving this are then in 1-1 correspondence with parallel symmetric cotractors in $\Gamma \mathcal{E}_{\left(A_{1} \cdots A_{k}\right)}$. The parallel tractor corresponding to a particular solution $\sigma$ is really part of the jet (roughly, Taylor series) data of $\sigma$ determined by prolongation (in [18] and cf. [5]), but remarkably we can avoid any significant details of this beyond what is implicit in the treament above.

We now see a generalisation of the Proposition above and corresponding refinement of Corollary 2.7. A first observation is that the tractor connection preserves a section of $\Lambda^{n+1} \mathcal{E}_{A}$ (a tractor volume form), and it follows that, without loss of generality, we may work with covariant tractor fields, and we henceforth make this simplification. However to work with these, and state results concisely, we shall need to recall some standard representation theory, as well as related notions and notation.

2.7. Tensors and representations. Up to isomorphism, each irreducible representation of $G$ is described by a weight

$$
\underline{r}=\left(r_{1}, \cdots, r_{n}\right), \quad \text { where } \quad r_{1} \geq \cdots \geq r_{n} \geq 0,
$$

and $r_{i} \in \mathbb{Z}_{\geq 0}, i=1, \cdots, n$. Below we use the notation $|\underline{r}|:=\sum_{1}^{n} r_{i}$. Equivalently the representation (2.9) is given by a Young diagram where from the top, and proceeding down, the rows have respective lengths $r_{1}, \cdots, r_{n}$, see e.g. [25]. As a shorthand for the weights we shall omit any terminal string of 0 s. For exmple $(2,2)$ means

$$
\underbrace{(2,2,0, \cdots, 0)}_{n} \text {. }
$$

Definition. $G$-type and $P$-type. Let us view $\left(\mathbb{R}^{n+1}\right)^{*}$ as the dual of the defining representation of $S L(n+1, \mathbb{R})$. $G$ and its subgroups act on the tensor algebra of $\left(\mathbb{R}^{n+1}\right)^{*}$ and tensors will be said to be of the same $G$-type (respectively $P$-type) if they lie in the same $G$-orbit (respectively $P$-orbit).

Each tensor power of $\bigotimes^{|\underline{x}|}\left(\mathbb{R}^{n+1}\right)^{*}$ may be decomposed into irreducible representations classified by the weights as in (2.9). Realising the irreducible representations in tensor powers of the (dual to the) standard representation is not unique. Certainly a tensor belonging to the representation (2.9) has valence (i.e. total rank) $|\underline{r}|:=\sum_{1}^{n} r_{i}$. The representation given in (2.9) will be realised by tensors

$$
I_{A_{1} \cdots A_{r_{1}} B_{1} \cdots B_{r_{2}} \cdots E_{1} \cdots E_{r_{n}}}
$$

which are completely symmetric over each of the respective index sets $A_{1} \cdots A_{r_{1}}, B_{1} \cdots B_{r_{2}}$, and so on to $E_{1} \cdots E_{r_{n}}$. We call a tensor in such a subrepresentation $G$-irreducible. The irreducibility of the representation is further encoded in what are sometimes termed hidden symmetries of the tensor elements [44]. For example symmetrising over any $r_{1}+1$ indices will annihilate the tensor $I$. With this understood we shall write $\mathbb{R}(\underline{r})$ for the vector space of such tensors in $\bigotimes^{\mid \underline{|r|}}\left(\mathbb{R}^{n+1}\right)^{*}$.

Finally as a point of notation. Above we have expressed the tensor $I$ as an object adorned with abstract indices. $\left(\mathbb{R}^{n+1}\right)^{*}$ has a standard basis, this generates a standard vectorial basis for the tensor algebra it generates. In terms of this we may express $I$ in terms of its components, and write

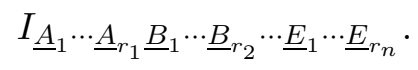


2.8. $G$-irreducible polynomial systems. As above, let us write $X^{\underline{A}}, \underline{A}=0,1, \cdots, n$ for the standard coordinates on $\mathbb{R}^{n+1}$. Given a tensor in $\bigotimes^{|\underline{x}|}\left(\mathbb{R}^{n+1}\right)^{*}$ we may construct polynomial systems by contraction in the obvious way. For example if $R_{A_{1} A_{2} B_{1} B_{2}} \in$ $\mathbb{R}(2,2)$ then we may form the two (in general non-trivial) polynomial systems

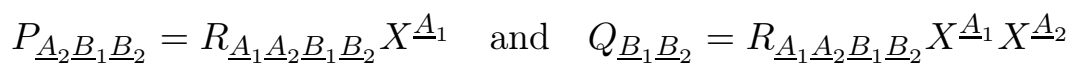

where repeated indices are summed (according to the Einstein summation convention). We shall term the latter of these saturated since any contraction of $X \underline{C}$ into $Q_{\underline{A B}}$ will result in anihilation, as a result of the (hidden) symmetries of $R$. Such polynomials form a natural class for many purposes, as shall be clear shortly.

Definition: We shall say that a polynomial system is $G$-irreducible if it arises as

$$
Q_{\underline{B}_{1} \cdots \underline{B}_{r_{2}} \cdots \underline{E}_{1} \cdots \underline{E}_{r_{n}}}=I_{\underline{A}_{1} \cdots \underline{A}_{r_{1}} \underline{B}_{1} \cdots \underline{B}_{r_{2}} \cdots \underline{E}_{1} \cdots \underline{E}_{r_{n}}} X^{\underline{A}_{1} \cdots X^{\underline{A}_{r_{1}}}}
$$

for some tensor $I \in \mathbb{R}(\underline{r})$. Note that the system is homogeneous of degree $r_{1}$, and defines a projective algebraic set on $\mathbb{S}^{n}$.

The system $Q$ given in (2.10) is saturated and homogeneous. By construction, as a tensor field on $\mathbb{R}^{n+1}$ it has symmetries consistent with the representation $\underline{r}^{\prime}=\left(r_{2}, \cdots, r_{n}\right)$. It now follows that these collectively imply that it corresponds to a certain field $\underline{\tau}$ on $\mathbb{S}^{n}$. In fact from (2.2) and the relationship between tractors bundle sections and cone tensor fields, as described in Section 2.4, we obtain that this tensor $\underline{\tau}$ is a section of weighted irreducible tensor bundle $\mathcal{E}\left(r_{2}, \cdots, r_{n}\right)(k)$, where $k=|\underline{r}|$. Here $\mathcal{E}\left(r_{2}, \cdots, r_{n}\right)$ is the bundle of covariant tensors having the Young symmetry $\left(r_{2}, \cdots, r_{n}\right)$ and $\mathcal{E}\left(r_{2}, \cdots, r_{n}\right)(k)=$ $\mathcal{E}\left(r_{2}, \cdots, r_{n}\right) \otimes \mathcal{E}(k)$. We shall say that a tensor field on $\mathbb{S}^{n}$ that arises in this way is a $G$-irreducible polynomial tensor field. The critical point here is that we can give a precise differential characterisation of these, that we shall come to now.

By their definition (and with Proposition 2.1) we see the weighted tensor bundles $\mathcal{E}\left(r_{2}, \cdots, r_{n}\right)(k), k \geq r_{2}+\left|r^{\prime}\right|$ in $\mathbb{Z}$, are exactly the bundles $\mathcal{B}^{0}$ in the BGG complexes mentioned in the introduction (see (1.2) ). Now we can state the full extension of Proposition 2.8 .

Proposition 2.9. The G-irreducible polynomial tensor fields on $\mathbb{S}^{n}$ are precisely the solutions of first $B G G$ operators $D: \mathcal{B}^{0} \rightarrow \mathcal{B}^{1}:$ If $\mathcal{B}^{0}$ is realised as the irreducible weighted tensor bundle $\mathcal{E}\left(r_{2}, \cdots, r_{n}\right)(k)$ and a section $\tau$ thereof is a solution (i.e. $D \tau=0$ ), then the homogeneous polynomial system corresponding to $\tau$ is the saturate of some $I \in \mathbb{R}\left(r_{1}, \cdots, r_{n}\right)$ where $r_{1}=k-\left|\underline{r}^{\prime}\right|$.

This result is an easy consequence of the main Theorem 2.10, which follows shortly.

Remark: Note that on $\mathbb{S}^{n}$ the homogeneous coordinates $X^{0}, \cdots, X^{n}$ linearly generate the full solution space of the $k=1$ system (1.1). Thus via the Proposition, these polynomially generate all first BGG solutions. The Proposition also gives the specific polynomial systems involved.

2.9. The fundamental theorem of normal solutions. As observed above each domain bundle $\mathcal{B}^{0}$, for a first BGG operator, may be realised in the form $\mathcal{E}\left(r_{2}, \cdots, r_{n}\right)(k)$. Let $\tau \in \Gamma \mathcal{E}\left(r_{2}, \cdots, r_{n}\right)(k)$ be a normal solution. Then $\tau=\Pi\left(I^{\tau}\right)$ where $I^{\tau}$ is a $G$ irreducible parallel tractor field. It is an easy consequence of the filtration structure arising from (2.2) that $\Pi$ may be realised explicitly by saturating $I^{\tau}$ with $X$ to yield

$$
Q_{B_{1} \cdots B_{r_{2}} \cdots E_{1} \cdots E_{r_{n}}}^{\tau}=I_{A_{1} \cdots A_{r_{1}} B_{1} \cdots B_{r_{2}} \cdots E_{1} \cdots E_{r_{n}}}^{\tau} X^{A_{1}} \cdots X^{A_{r_{1}}},
$$


(where $r_{1}=k-\left|\underline{r^{\prime}}\right|$ ) and by then contracting the projectors $Z_{a}^{A}$ (of $(\underline{2.6})$ ) onto all remaining indices. Note that $Q^{\tau}$ takes values in an irreducible subbundle of the weighted tractor bundle $\mathcal{E}_{B_{1} \cdots B_{r_{2}} \cdots E_{1} \cdots E_{r_{n}}}\left(r_{1}\right)$ and the final step of contracting with the concatenation of $Z^{A}{ }_{a}$ projectors is simply realising the isomorphism between this subbundle and the weighted tensor bundle $\mathcal{E}\left(r_{2}, \cdots, r_{n}\right)(k)$. Thus we henceforth identify $\mathcal{E}\left(r_{2}, \cdots, r_{n}\right)(k)$ with this subbundle (as done implicitly in Corollary 2.7) and thus $\tau$ and $Q^{\tau}$ are also to be identified.

Now fix an arbitrary point $\tilde{q} \in \boldsymbol{M}$. In a neighbourhood of $\tilde{q}$, and in terms of a normal frame field (as defined in (2.8), and see the Remark below that) we have

$$
Q_{\underline{B}_{1} \cdots \underline{B}_{r_{2}} \cdots \underline{E}_{1} \cdots \underline{E}_{r_{n}}}^{\tau}=I_{\underline{A}_{1} \cdots \underline{A}_{r_{1}} \underline{B}_{1} \cdots \underline{B}_{r_{2}} \cdots \underline{E}_{1} \cdots \underline{E}_{r_{n}}} X^{\underline{A}_{1} \cdots X^{-} \underline{A}_{r_{1}}}
$$

and, by Corollary 2.6, the components $I_{\underline{A}_{1} \cdots \underline{A}_{r_{0}} \underline{B}_{1} \cdots \underline{B}_{r_{1}} \cdots \underline{E}_{1} \cdots \underline{E}_{r_{n}}}$ are constant. Using again Corollary 2.6 to interpret this on $\mathbb{S}^{n}$ we have the following.

Theorem 2.10. Suppose that $\tau \in \Gamma \mathcal{E}\left(r_{2}, \cdots, r_{n}\right)(k)$ is a normal solution of the equation $D \tau=0$ on $\left(M^{n}, p\right)$. For an arbitrary point $q \in M$, fix a an adapted frame at $q$. This determines a normal frame $f_{\underline{A}}$ (as in [2.8), a local diffeomorphism $\phi: \mathbb{S}^{n} \rightarrow M$, and corresponding generalised homogeneous coordinates $X^{\underline{A}}$ in a neighbourhood of $q$. In terms of these the following hold:

- With respect to the trivialisation of $\mathcal{E}_{B_{1} \cdots B_{r_{2}} \cdots E_{1} \cdots E_{r_{n}}}\left(r_{1}\right)$, determined by $f_{\underline{A}}$, the coordinate functions $\underline{\tau}$ (of $\tau$ ) form a homogeneous polynomial system in the generalised homogeneous coordinates.

- The collection $\Phi^{*} \underline{\tau}$ is given by the same formal polynomial system, where the $X \underline{A}$ are now interpreted as the standard coordinates of $\mathbb{R}^{n+1}$. With respect to the standard frame on $\mathbb{R}^{n+1}$, the collection $\Phi^{*} \underline{\tau}$ are the coordinate functions of a solution $\tau^{\prime}$ of the equation $D \tau^{\prime}=0$ on $\mathbb{S}^{n}$.

Remark: Note that the polynomial system $\underline{\tau}$, as in the Theorem, satisfies polynomial relations. These arise in an obvious way from the fact that tractor section, equivalent to $\tau$, is saturated with respect to contraction with $X^{A}$. For example in the case $\tau \in \Gamma \mathcal{E}_{a}(2)$ then the system $\underline{\tau}$ consists of the $n+1$ linear polynomials $K_{\underline{A B}} X \underline{B}$ where the matrix of components of $\bar{K}$ is skew, i.e. $K_{\underline{A B}}=-K_{\underline{B A}}$. Thus there is the one polynomial relation $K_{\underline{A B}} X \underline{A} X \underline{B}=0$.

Next note that since the reasoning in the first part of the proof above applies, in particular, when we begin with $\tau$ a solution of $D$ in the "flat case" (i.e. on $\mathbb{S}^{n}$ with its standard projective structure) as a corollary we have at once the Proposition 2.9.

2.10. The zero locus of normal solution. The Theorem above is local in nature but it (or equivalently Corollary 2.7) has a global interpretation. Before we come to this we need some simple observations, and a definition.

Definition. G-type and P-type of a point. Consider a projective manifold $(M, p)$ equipped a with a normal solution $\tau$, and let $I^{\tau}$ be the parallel tractor such that $\tau=\Pi\left(I^{\tau}\right)$. For a point $q \in M$ we can choose a tractor frame $e_{\underline{A}}$ for the tractor space $\mathcal{T}_{q}$ at $q$, which is adapted in the sense that $e_{0}$ is parallel to $X^{A}$. Using this frame, the components of $I^{\tau}(q)$ define an element in the tensor algebra of $\left(\mathbb{R}^{n+1}\right)^{*}$. As we have noted in 2.3 any two such frames are related by the action of an element of $P$. Hence the $P$-orbit of this element, which we call the P-type of the point $q$, depends only on $(M, p)$, $\tau$, and $q$, and not on further choices. Of course, this implies that also its $G$-orbit is well defined, and we call this the G-type of the point $q$ 
Since a parallel tractor has constant components with respect to the normal frames $f_{\underline{A}}$ which, although not adapted, are volume mormalised, we obtain the following.

Theorem 2.11. If $(M, p)$ is connected then any parallel tractor field I has a constant G-type.

An analogous statement is not available for $P$-type, because an adapted frame is not parallel along any curve; this is clear from (2.7).

It follows from Theorem 2.11 that, for a normal solution $\tau$ on a connected manifold $(M, p)$, we may associate a fixed $G$-irreducible polynomial tensor field $\tau^{\prime}$ on the model $\mathbb{S}^{n}$, and this is obviously unique (up to a $G$-action that we shall ignore). In this case we shall say that $\left(\mathbb{S}^{n}, \tau^{\prime}\right)$ is the model for $(M, p, \tau)$ (meaning $(M, p)$ equipped with a normal solution $\tau$ ).

For a given normal solution $\tau$ on a projective manifold $(M, p)$, its zero locus $\mathcal{Z}(\tau)$ is not necessarily smooth. However, given $q \in \mathcal{Z}(\tau)$, Corollary 2.7 shows that there is a point $q^{\prime} \in \mathcal{Z}\left(\tau^{\prime}\right) \subset \mathbb{S}^{n}$ and a local diffeomorphism from an open neighborhood of $q$ to an open neighborhood of $q^{\prime}$ in the model, which is compatible with resepect to the zero sets. In particular, $\mathcal{Z}(\tau)$ cannot have worse singularities than $\mathcal{Z}\left(\tau^{\prime}\right)$. For example if $q^{\prime}$ is a smooth point then, in a neighbourhood of $q, \mathcal{Z}(\tau)$ is an embedded smooth submanifold. Hence the problem of the classification of zero locus singularities for normal solutions is reduced to a problem in real algebraic geometry. Note that it can be that the model algebraic set is not (globally) smooth and yet the zero locus $\mathcal{Z}(\tau)$ is smooth and embedded; indeed as an extreme case $\mathcal{Z}(\tau)$ may be empty.

For emphasis we collect some of these definitions and consequences of Theorem 2.10 into a statement.

Corollary 2.12. Let $\tau$ be a normal solution on a connected manifold $(M, p)$ and let $\left(\mathbb{S}^{n}, \tau^{\prime}\right)$ be the corresponding model. If $q \in \mathcal{Z}(\tau)$ then there some $q^{\prime} \in \mathcal{Z}\left(\tau^{\prime}\right)$ with the same singularity type. In particular, if $\mathcal{Z}\left(\tau^{\prime}\right)$ is a smooth algebraic set then $\mathcal{Z}(\tau)$ is a smooth embedded submanifold.

2.11. Orbit type decomposition of $M$. Here we observe that a normal solution $\tau$ (equivalently a parallel tractor field $I^{\tau}$ ) determines a canonical stratification of $M$.

Different points on the manifold may have the same $P$-type and this establishes an equivalence relation for the points of $M$. Thus the points of the manifold $M$ are partitioned according to $P$-type. On a given structure $(M, p, \tau)$ there can be many orbit types. For example a point where $\tau$ vanishes is certainly in a different $P$-type to a point where $\tau$ is not zero. In general the $P$-type decomposition exposes considerably finer structure than this example illustrates. We treat some examples in Section 3.1 below, but a more detailed analysis is the subject of [15].

Now we claim that the diffeomorphism $\phi$, of Lemma 2.4. preserves $P$-type. Precisely, the $P$-type of $I^{\tau}$ at $\phi(y)$ coincides with the $P$-type of $I^{\tau^{\prime}}$ at $y$. To see this, arguing as in the proof of Theorem [2.5, simply observe that these tractors have the same coordinate expression in different frames, both of which are adapted. This exactly means same they have the same $P$-type.

Thus we may take the alternative view that the maps $\phi$ transfers the $P$-type decomposition of the model $\left(\mathbb{S}^{n}, \tau^{\prime}\right)$ onto the corresponding curved structure $(M, p, \tau)$. Part of the power of this lies in the following result (discussed in greater detail in [15]). 
Proposition 2.13. Let $G_{I^{\prime}} \subset G$ be the isotropy subgroup of a tensor $I^{\prime}$ in $\otimes \mathbb{R}^{*}$. Viewing $I^{\prime}$ as a parallel tractor on $\mathbb{S}^{n}$, the P-type decomposition of $\left(\mathbb{S}^{n}, I^{\prime}\right)$ is the same as the orbit decomposition of $\mathbb{S}^{n}$ under the action of $G_{I^{\prime}}$.

Proof: It is easily verified directly, that in either case the orbits are naturally parametrised by the points of the double coset space $G_{I^{\prime}} \backslash G / P$.

In general a projective manifold $(M, p)$, with a parallel tractor $I$, admits no action by

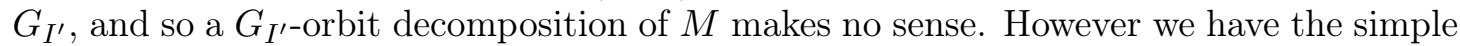
but surprising outcome that the $P$-type decomposition is well defined, and this echoes the $G_{I^{\prime}}$-orbit decomposition of the model. For example it follows from the Proposition 2.13 that the $P$-types of $\mathbb{S}^{n}$ are non-self-intersecting smoothly immersed (in fact initial [37, Theorem 5.14]) submanifolds and hence, via the diffeomorphism $\phi$ of Lemma 2.4, we may conclude that so are the $P$-types of $(M, p, I)$. Thus the $P$-types give a smooth stratification of $M$. In summary we have the following.

Theorem 2.14. Let $(M, p)$ be projective manifold equipped with a parallel tractor $I$. Then $(M, p)$ is stratified according to a P-type decomposition. The diffeomorphism $\phi$ of Lemma 2.4 preserves the $P$-type and, in particular, the occurring $P$-types are a selection of those arising in the model $\left(\mathbb{S}^{n}, I^{\prime}\right)$.

2.12. Geometry. The presence of a parallel tractor equips $(M, p)$ with additional geometric structure. Of course a parallel tractor may be reinterpreted as a reduction of the (projective) tractor holonomy. By definition this is additional geometric structure, and as mentioned in the introduction, local and generic aspects of this have been explored in, for example, [3, 4]. In the examples of the next section we shall see, from our current point of view, how such classical structures arise. In one case we shall see the structure is necessarily non-Ricci-flat pseudo-Riemannian Einstein on open subsets.

More importantly for the directions here, we show that the parallel tractor, along with Theorems 2.5 and 2.10, provides a tool which can relate the geometry of a normal solution zero locus to that on the complementary space.

\section{EXAMPLES}

3.1. Preliminary observations. In several of the examples below we consider normal solutions $\tau$ which are sections of a density bundle $\mathcal{E}(w)$, where $w \neq 0$, and the open $P$ types are submanifolds on which $\tau$ is non-vanishing. On such a $P$-type, denoted by $M_{+}$ say, $\tau$ is a scale and so naturally determines an affine connection $\nabla^{\tau}$ from the projective class (restricted to $M_{+}$) as discussed in Section 2.2. We want to explain how our method can be used to prove results related to geodesic completeness of $\nabla^{\tau}$. Consider a geodesic path in $M$ which leaves $M_{+}$, i.e. which intersects both $M_{+}$and $\mathcal{Z}(\tau)$. Take a point $q \in \mathcal{Z}(\tau)$ which lies in the closure of $M_{+}$and carry out the construction from Lemma 2.4 for some point $\tilde{q}$ over $q$. Then our geodesic path will become a geodesic $\gamma$ for the connection determined by the normal scale $\sigma$ determined by the construction.

Now take a point $x \in M_{+}$which lies on $\gamma$ (and in the range of the diffeomorphism $\phi$ ). Starting from $x$ and moving along $\gamma$ in the direction of $q$, the point $q$ will of course be reached in finite time. Now some reparametrization $\hat{\gamma}$ of $\gamma$ will be a geodesic for $\nabla^{\tau}$, and it may happen that this reparametrization has the effect that $q$ is no longer reached in finite time.

If we assume that the original manifold $M$ is closed then the only way for $M_{+}$to be geodescially incomplete is that geodesics leave $M_{+}$in finite time. Hence if in the above considerations $q$ is never reached in finite time, geodesic completeness follows. 
The reparametrization from $\gamma$ to $\hat{\gamma}$ can be obtained as the solution of a an ODE which depends only on the function describing $\tau$ in the trivialization of the density bundle determined by $\sigma$. But now it follows from the construction that the diffeomorphism $\phi$ relates $\sigma$ and the function describing $\tau$ in the trivialization determined by $\sigma$ to their counterparts on the homogeneous model. Consequently, the reparametrizations on the homogeneous model and on the curved manifold are determined by the same ODE and hence coincide. Thus, if the point $\phi^{-1}(q)$ on the homogeneous model is not reached in finite time after the reparametrisation, then the same is true for $q$. In particular, geodesic completeness carries over from the homogenous model to curved geometries on closed manifolds.

If in such a situation $\mathcal{Z}(\tau)$ is the boundary of $M_{+}$(as in first two examples below) then it reasonable to call $\mathcal{Z}(\tau)$ the projective infinity for $\left(M_{+}, \nabla^{\tau}\right.$ ) (as an analogue of the term conformal infinity). This is not meant to imply that that $\mathcal{Z}(\tau)$ necessarily has a canonical projective structure.

3.2. The parallel standard cotractor - projective almost Ricci-flat. Here we obtain a structure which generalises that of an affine Ricci flat manifold (cf. [45]); the result is affine Ricci-flat on an open dense set. From the point of view of compactifications, it yields a structure that is a compactification of a Ricci-flat manifold that is a curved generalisation of the usual projective compactification of the affine plane to a hemisphere via central projection. Thus, in particular, it is different to a conformal compactification of such a space (which for the case of the Euclidean plane is a 1-point compactification).

The structure in this case in a projective manifold $(M, p)$ equipped with a parallel section $I_{A}$ of the standard cotractor bundle. Let $\sigma$ denote $I_{A} X^{A}$. To find the first BGG equation that this satisfies we may calculate with respect to $\nabla \in p$. We have $I_{B} \stackrel{\nabla}{=}\left(\mu_{b} \sigma\right)$ and using the formula for the tractor connection (2.3), we see that $I_{B}$ parallel implies that $\mu_{b}=\nabla_{b} \sigma$ and then

$$
\nabla_{a} \nabla_{b} \sigma+P_{a b} \sigma=0
$$

this is the $k=1$ case of (1.1). All solutions of (3.1) arise this way (i.e. any solution of (3.1) is normal), indeed a prolongation of this equation determines the tractor connection [6].

Where $\sigma$ is non-vanishing (3.1) is the equation that $\nabla$ is projectively Ricci-flat; more precisely in an open neighbourhood with $\sigma$ nowhere zero the connection $\widehat{\nabla}$, characterised by $\widehat{\nabla} \sigma=0$ and hence related to $\nabla$ via (2.1) with $\Upsilon_{a}$ given by $\sigma^{-1} \nabla_{a} \sigma$, is Ricci flat. Thus the structure $\left(M, p, I_{A}\right)$ generalises the notion of a Ricci-flat affine manifold.

The model structure is $\left(\mathbb{S}^{n}, \sigma^{\prime}\right)$ where $\sigma^{\prime}$ is the weight 1 density which arises as a projective polynomial from $\boldsymbol{I}_{\underline{A}} X^{\underline{A}}$ on $\mathbb{R}^{n+1}$ where $\boldsymbol{I}_{\underline{A}}$ is a constant covector there. Since $\boldsymbol{I}_{\underline{A}} X^{\underline{A}}=0$ describes a hyperplane through the origin in $\mathbb{R}^{n+1}$ it follows that the zero locus of $\sigma^{\prime}$ is a totally geodesic embedded $\mathbb{S}^{n-1}$ in $\mathbb{S}^{n}$ with its standard projective structure. The $P$-type decomposition consists of the 3 submanifolds where $\sigma^{\prime}$ is, respectively, positive, zero, and negative. On the open submanifolds $\mathbb{S}_{ \pm}^{n}$ where $\sigma^{\prime}$ is, respectively, positive, and negative, $\sigma^{\prime}$ is a scale and induces the flat connection $\nabla^{\sigma^{\prime}}$ in agreement with the identification (by central projection) of $\mathbb{S}_{ \pm}^{n}$ with, respectively, the affine subspaces in $\mathbb{R}^{n+1}$ descibed by $\boldsymbol{I}_{\underline{A}} X^{\underline{A}}= \pm 1$. Thus these manifolds $\left(\mathbb{S}_{ \pm}^{n}, \nabla^{\sigma^{\prime}}\right)$ are geodesically complete. According to our general results above these features are necessarily repoduced in the general situation. Thus we have the following. 
Theorem 3.1. Consider a projective manifold $(M, p)$ equipped with a parallel standard cotractor $I_{A}$. Then the weight 1 projective density $\sigma=I_{A} X^{A}$ satisfies the equation (3.1). The manifold is stratified by $P$-types $M_{+}, M_{o}, M_{-}$according to the strict sign of $\sigma$. These components have a structure as follows:

- The zero locus $M_{o}$ of $\sigma$ is either empty, or forms a smooth embedded hypersurface. With respect to any $\nabla \in p$, this is totally geodesic, and has canonically an intrinsic projective structure $p^{M_{o}}=\left[\nabla^{M_{o}}\right]$ where $\nabla^{M_{o}}$ is simply the restriction of $\nabla$. The normal tractor connection of $\left(M_{o}, p^{M_{o}}\right)$ is naturally a restriction of the ambient tractor connection.

- The open submanifolds $\left(M_{ \pm}, \nabla^{\sigma}\right)$ are Ricci-flat affine manifolds, which are geodesically complete if $M$ is closed.

- If $M \backslash M_{+}$or $M \backslash M_{-}$is compact (e.g. if $M$ is closed this is forced) then $\left(M \backslash M_{\mp}, p, I\right)$ is a geometrically canonical compactification of, respectively, $\left(M_{ \pm}, p\right)$ (where throughout $p$ and $I$ are restricted to the indicated submanifolds).

Proof: The last bullet point is simply the observation that the construction yields a projective analogue of conformal compactification of Einstein manifolds.

Concerning the zero locus: On the model the zero locus is a totally geodesic equatorial embedded $(n-1)$-sphere. It follows at once that $M_{o}$ is totally geodesic, as based around a point $q$ in $\mathcal{Z}(\sigma)$, by construction $\Phi^{-1}$ is compatible with the geodesic paths through $q$. Thus $M_{o}$ has a projective structure which is simply a restriction of that from the ambient $(M, p)$.

This result can also be seen via the Thomas cone space since there $\boldsymbol{I}_{A}$ is parallel, and thus is a conormal to the zero locus of $\boldsymbol{\sigma}=\boldsymbol{I}_{A} \zeta^{A}$ (where we have used $\boldsymbol{\nabla}_{A} \zeta^{B}=\delta_{A}^{B}$ ). Since this zero locus $\mathcal{Z}(\boldsymbol{\sigma})$ has a parallel conormal it is totally geodesic. On the other hand the non-vertical geodesics of $\boldsymbol{M}$ are the lifts of the geodesics from $(M, p)$, and it follows that $M_{o}$ is totally geodesic. Using now that $\mathcal{Z}(\boldsymbol{\sigma})$ is totally geodesic, it follows that it inherits an affine manifold structure by the restriction of the ambient $\nabla$. The claims about the normal tractor connection follow, with this restriction of $\boldsymbol{\nabla}$ to $\mathcal{Z}(\boldsymbol{\sigma})$ being the Thomas space over $\left(M_{o},\left.p\right|_{M_{o}}\right)$.

Away from its zero locus, $\sigma$ is a scale and so we have $\nabla^{\sigma} \sigma=0$, thus the claim that the components $\left(M_{ \pm}, \nabla^{\sigma}\right)$ are Ricci-flat follows from (3.1).

All other points follow immediately from the corresponding results on the model via Theorem 2.10 (and its proof), Corollary 2.12, Theorem 2.14, and the discussion of section 3.1

3.3. A parallel tractor metric - Klein-Einstein structures. Here we consider a projective manifold $(M, p)$ equipped with a non-degenerate symmetric and parallel 2cotractor $H_{A B}$ of signature $(r, s), r \geq s \geq 0$. In this case $\sigma:=H_{A B} X^{A} X^{B}$ satisfies the third order equation

$$
\nabla_{(a} \nabla_{b} \nabla_{c)} \sigma+4 P_{(a b} \nabla_{c)} \sigma+2\left(\nabla_{(a} P_{b c)}\right) \sigma=0
$$

where $(\cdots)$ indicates the symmeytric part over the enclosed indices.

The model structure is $\left(\mathbb{S}^{n}, \sigma^{\prime}\right)$ where $\sigma^{\prime}$ is the weight 2 density which arises as a polynomial scalar density from the homogeneous polynomial $\boldsymbol{\sigma}:=\boldsymbol{H}_{\underline{A B}} X^{\underline{A}} X \underline{B}$ on $\mathbb{R}^{n+1}$, where $\boldsymbol{H}_{A B}$ is a fixed (signature $(r, s)$ ) inner product there.

If $s \geq 1$ then $\boldsymbol{H}_{\underline{A B}} X^{\underline{A}} X^{\underline{B}}=0$ is a quadratic variety in $\mathbb{R}^{n+1}$ and, corresponding to this, the zero locus of $\sigma^{\prime}$ is an embebbed variety $S^{r-1} \times S^{s-1}$ in $\mathbb{S}^{n}$ with a signature $(r-1, s-1)$ conformal structure induced from $\boldsymbol{H}_{\underline{A B}}$ (viewed now as a metric in $\mathbb{R}^{n+1} \backslash\{0\}$ ) restricted to tangent vectors in $\mathcal{Z}(\boldsymbol{\sigma})$. The $P$-type decomposition consists of the 3 submanifolds where $\sigma^{\prime}$ is, respectively, positive, zero, and negative. On the open submanifolds $\mathbb{S}_{ \pm}^{n}$ 
where $\sigma^{\prime}$ is, respectively, positive, and negative, $\sigma^{\prime}$ is a scale and induces a spaceform metric, with signature respectively $(r, s-1)$ and $(r-1, s)$, and with $\nabla^{\sigma^{\prime}}$ the compatible Levi-Civita connection having curvature, respectively, negative and positive. It is well known that these that these manifolds $\left(\mathbb{S}_{ \pm}^{n}, \nabla^{\sigma^{\prime}}\right)$ are geodesically complete.

If $s=0$ then the model is very simple. Then $\mathcal{Z}(\boldsymbol{\sigma})$ is empty on $\mathbb{R}^{n+1} \backslash\{0\}$. There is just the one $P$-type, viz. $\mathbb{S}^{n}$, and, via $\sigma, H_{A B}$ induces the usual (up to diffeomorphism) unit round metric on this, and $\nabla^{\sigma}$ is the standard Levi-Civita connection. In general we have the following.

Theorem 3.2. Consider a projective manifold $(M, p)$ equipped with a non-degenerate symmetric and parallel 2-cotractor $H_{A B}$ of signature $(r, s), r \geq s \geq 0$. The weight 2 projective density $\sigma=H_{A B} X^{A} X^{B}$ satisfies the equation (3.2). If $s=0$ then:

- $M$ is a single $P$-type and $\sigma$ is a scale on $M$ that induces a positive Einstein metric. If $s \geq 1$ we have the following:

- The manifold is stratified by P-types $M_{+}, M_{o}, M_{-}$according to the strict sign of $\sigma$.

- The zero locus $M_{o}$ of $\sigma$ is either, empty, or forms a smooth embedded hypersurface with a conformal structure $c$ of signature $(r-1, s-1)$. The standard conformal tractor bundle agrees with the restriction of the projective tractor bundle $\mathcal{T}$ to $M_{o}$ and the normal conformal tractor connection of $\left(M_{o}, c\right)$ is naturally the corresponding restriction of the ambient projective tractor connection.

- On the open submanifold $M_{ \pm}, \sigma$ is a scale that induces, respectively, a positive/negative Einstein metric $g^{\sigma}$ of signature $(r-1, s)$ or $(r, s-1)$. In each case, the affine connection $\nabla^{\sigma}$ is the corresponding Levi-Civita connection. If $M$ is closed then each of $\left(M_{ \pm}, g^{\sigma}\right)$ is geodesically complete.

- If $M \backslash M_{+}$or $M \backslash M_{-}$is compact with boundary $M_{o}$ (e.g. if $M$ is closed this is forced) then $\left(M \backslash M_{\mp}, p, H\right)$ is a geometrically canonical compactification of, respectively, $\left(M_{ \pm}, g^{\sigma}\right)$ ( $H$ is restricted to the indicated submanifolds).

Proof: Since a scale trivialises the bundles $\mathcal{E}(w)$ and splits the dual Euler sequence (2.5), it follows that in the presence of a scale, $H_{A B}$ determines a covariant symmetric two tensor on the manifold $M$. As in the case of the model, the signature of this depends on the $P$-type, and indeed via the diffeomorphism $\Phi$, of Lemma 2.4, we can conclude the signature of each $P$-type from the model; in either case this is determined in an obvious way according to whether $X^{A}$ is timelike or spacelike with respect to $H_{A B}$. Calculating locally where $\sigma$ is a scale one sees that $\nabla_{a}^{\mathcal{T}} H_{B C}=0$ implies that $\nabla_{a}^{\sigma} P_{b c}=0$ and that $P_{b c}$ agrees up to a constant (giving the sign of the scalar curvature) with the metric induced from $H_{B C}$, and this constant is non-zero on the open $P$-types [3, 31]. Since $\nabla^{\sigma}$ is torsion-free it follows that on these $P$-types it is an Einstein Levi-Civita connection.

$M_{O}$ is the set where $X^{A}$ is null (with respect to $H_{A B}$ ). But, where $X^{A}$ is null, one easily sees that $H_{A B}$ determines a signature $(r-1, s-1)$ bilinear form, taking values in $\left.\mathcal{E}(2)\right|_{M_{o}}$, that is independent of any splitting of (2.5). This is locally compatible with the model via $\Phi$. Since $H_{A B}$ is parallel, this is in particular so along $M_{o}$, and so $\nabla^{\mathcal{T}}$ is metric preserving along $M_{o}$. Here, as elsewhere, we have that in any choice of weight 1 scale $\sigma$, and with $\nabla^{\sigma}$ for the moment denoting the coupled scale-tractor connection, we have that $\nabla_{a}^{\sigma}\left(\sigma^{-1} X^{B}\right)$ gives a splitting of (2.5) . Combining with the fact that $\nabla^{\mathcal{T}}$ is torsion free it follows, using its characterisation in [12], that $\nabla^{\mathcal{T}}$ agrees with the normal conformal tractor connection along $M_{o}$.

As in the previous example, all remaining facts follow immediately from the corresponding results on the model via Theorem 2.10, Corollary 2.12, Theorem 2.14, and the discussion of section 3.1 . 
Remarks: The case that $H_{A B}$ has Lorentzian signature is important. Looking at the model $\left(\mathbb{S}^{n}, \sigma^{\prime}\right)$, the part $\mathbb{S}_{-}^{n}$ where $\sigma^{\prime}$ is negative consists of two copies of hyperbolic space $\mathbb{H}^{n}$ antipodally placed as the interior of the standard double $S^{n-1}$ quadric on $\mathbb{S}^{n}$. The compactification $\mathbb{S}^{n} \backslash \mathbb{S}_{+}^{n}$ adds the boundary spheres. Since this model is based on central projection (with e.g. geodesics arising from planes through the origin) it is natural to think of the result as two copies of the Klein model of $\mathbb{H}^{n}$; whence the curved analogue could be called a Klein-Einstein manifold by analogy with the use of the term Poincare-Einstein in the literature. Note that the conformal structure of the interior of the Klein-Einstein (KE) manifold does not extend to the boundary, even though the latter has a canonical conformal structure. This is clear by continuity considerations, for example, since the signature of the ambient metric changes as we cross the zero locus of $\sigma$. Thus we have a result, which we state as proposition in order to highlight.

Proposition 3.3. A Klein-Einstein manifold involves a compactification of its Einstein interior that is strictly different to the conformal compactification of a Poincaré-Einstein (PE) manifold; there is never a smooth diffeomporphism between a PE manifold and KE manifold that restricts to a conformal map on the interior.

Returning to the model with $H_{A B}$ Lorentzian, the component $\mathbb{S}_{+}^{n}$ is the geometry known as de Sitter space in the general relativity literature; $\mathbb{S}^{n} \backslash \mathbb{S}_{-}^{n}$ is the projective compactification of this. Again $\left(M \backslash M_{-}, p, H_{A B}\right)$ is a curved analogue.

It is not difficult to show that the Klein-Einstein compactification described here is the same as the projectively compact (Einstein) metric described by Fefferman-Graham in [23, chapter 4]. There it is defined as a manifold with boundary equipped with a negative Einstein metric on the interior that near the boundary takes form $h / \rho+d \rho^{2} / 4 \rho^{2}$; here $\rho$ is a defining function for the boundary, while $h$ a symmetric 2-tensor $h$ which is smooth to the boundary, and with restriction there a signature $(p, q)$ boundary metric. As explained in [23], by an appropriate change of variables these structures may be transformed to Poincaré-Einstein manifolds satisfying an evenness condition, and hence are also closely related to the Fefferman-Graham ambient metric. Further details of the geometry of the Klein-Einstein type structures, and their links to PE manifolds, is taken up in [29, 31.

3.4. Singular and higher codimension zero locus. Examples with singular zero locus arise easily in the case where we assume more than one parallel tractor field. For example if we assume $I_{A}^{1}$ and $I_{A}^{2}$ are linearly independent parallel cotractor fields, on a given projective manifold $(M, p)$, then $S_{A B}:=I_{A}^{1} I_{B}^{2}+I_{B}^{1} I_{A}^{2}$ is symmetric and parallel. Thus we are in the situation of the previous example except that $S_{A B}$ is far from nondegenerate. We have

$$
\mathcal{Z}\left(X^{A} X^{B} S_{A B}\right)=\mathcal{Z}\left(\sigma^{1} \sigma^{2}\right)=\mathcal{Z}\left(\sigma^{1}\right) \cup \mathcal{Z}\left(\sigma^{2}\right),
$$

where $\sigma^{1}=I_{A}^{1} X^{A}$ and $\sigma^{2}=I_{A}^{2} X^{A}$. In the model, and generically, this is not smooth. There are three $P$-types according to whether none, one, or both of $\sigma^{1}$ and $\sigma^{2}$ is zero. Geometrically, on an open dense set, the structure $\left(M, p, S_{A B}\right)$ has projectively related (in the sense of (2.1)) Ricci-flat affine structures.

Assume $(M, p), I^{1}$, and $I^{2}$ as above, and set $K_{A B}=I_{A}^{1} I_{B}^{2}-I_{B}^{1} I_{A}^{2}$, then we generically obtain a smooth codimension 2 zero locus

$$
\mathcal{Z}\left(k_{a}\right)=\mathcal{Z}\left(\sigma^{1}\right) \cap \mathcal{Z}\left(\sigma^{2}\right)
$$

for the weight 2 one-form field $k_{a}=\sigma^{1} \nabla_{a} \sigma^{2}-\sigma^{2} \nabla_{a} \sigma^{1}$ which corresponds to $K_{A B} X^{B}$. (Here $\nabla$ is any connection from $p$ ). We have $K_{A B} X^{B}=Z_{A}^{b} k_{b}$ where $Z_{A}^{b}$ is the projectively 
invariant bundle monomorphism in the Euler sequence (2.2), and note that $\nabla_{a} \sigma^{i}$ is nonvanishing along $\mathbb{Z}\left(\sigma^{i}\right)$ as $I^{i} \neq 0$, for $i=1,2$. ) There are two $P$-types: simply $\mathcal{Z}\left(k_{a}\right)$ and its complement. Note that the first BGG equation in this case is

$$
\nabla_{(a} k_{b)}=0 .
$$

\section{REFERENCES}

[1] M.T. Anderson, Boundary regularity, uniqueness and non-uniqueness for AH Einstein metrics on 4-manifolds, Adv. Math. 179 (2003), 205-249.

[2] S. Armstrong, Definite signature conformal holonomy: a complete classification, J. Geom. Phys. 57 (2007), 2024-2048.

[3] S. Armstrong, Projective holonomy. I. Principles and properties, Ann. Global Anal. Geom., 33 (2008), 47-69.

[4] S. Armstrong, Projective holonomy. II. Cones and complete classifications, Ann. Global Anal. Geom. 33 (2008), 137-160.

[5] T. Branson, A. Čap, Eastwood, A.R. Gover, Prolongations of geometric overdetermined systems, Internat. J. Math. 17 (2006), 641-664.

[6] T.N. Bailey, M.G. Eastwood, and A.R. Gover, Thomas's structure bundle for conformal, projective and related structures, Rocky Mountain J. Math. 24 (1994), 1191-1217.

[7] I.N. Bernstein, I.M. Gelfand, S.I. Gelfand, Differential operators on the base affine space and a study of $\mathfrak{g}$-modules. Lie groups and their representations (Proc. Summer School, Bolyai János Math. Soc., Budapest, 1971), pp. 21-64.

[8] O. Biquard, Métriques d'Einstein asymptotiquement symétriques, Astérisque, No. 265 (2000), vi +109 pp.

[9] O. Biquard, R. Mazzeo, Parabolic geometries as conformal infinities of Einstein metrics, Arch. Math. (Brno) 42 (2006), suppl., 85-104.

[10] A. Chang, J. Qing, and P. Yang, Renormalized volumes for conformally compact Einstein manifolds, (Russian) Sovrem. Mat. Fundam. Napravl. 17 (2006), 129-142; translation in J. Math. Sci. (N.Y.) 149 (2008), 1755-1769

[11] A. Čap, and A.R. Gover, Tractor calculi for parabolic geometries, Trans. Amer. Math. Soc. 354 (2002), 1511-1548.

[12] A. Cap, and A.R. Gover, Standard tractors and the conformal ambient metric construction, Ann. Global Anal. Geom. 24 (2003), 231-295.

[13] A. Čap, and A.R. Gover, CR-Tractors and the Fefferman Space, Indiana University Mathematics Journal, 57 (2008), 2519-2570

[14] A. Čap, and A.R. Gover, A holonomy characterisation of Fefferman spaces, arXiv:math/0611939

[15] A. Čap, A.R. Gover, M. Hammerl, in progress.

[16] A. Čap, J. Slovák, Parabolic geometries. I. Background and general theory. Mathematical Surveys and Monographs, 154. American Mathematical Society, Providence, RI, 2009. x+628 pp.

[17] A. Čap, J. Slovák, V. Souček, Invariant operators on manifolds with almost Hermitian symmetric structures. III. Standard operators, Differential Geom. Appl. 12 (2000), 51-84.

[18] A. Čap, J. Slovák, V. Souček Bernstein-Gelfand-Gelfand sequences, Ann. of Math., (2) 154 (2001), 97-113.

[19] S.Y. Cheng, S.T. Yau, On the existence of a complete Kähler metric on noncompact complex manifolds and the regularity of Fefferman's equation, Comm. Pure Appl. Math 33 (1980), 507544.

[20] C. Fefferman, Monge-Ampère equations, the Bergman kernel and geometry of pseudoconvex domains, Ann. of Math. 103 (1976), 395-416; Erratum 104 (1976), 393-394.

[21] C. Fefferman, and C.R. Graham, Conformal invariants in: The mathematical heritage of Élie Cartan (Lyon, 1984). Astérisque 1985, Numero Hors Serie, 95-116.

[22] C. Fefferman, and C.R. Graham, Q-curvature and Poincaré metrics, Math. Res. Lett. 9 (2002), $139-151$.

[23] C. Fefferman, and C.R. Graham, The Ambient Metric, Princeton University Press, to appear. arXiv:0710.0919.

[24] D.J.F. Fox, Contact projective structures Indiana Univ. Math. J. 54 (2005), 1547-1598. 
[25] W. Fulton, J. Harris, Representation theory. A first course. Graduate Texts in Mathematics, 129. Readings in Mathematics. Springer-Verlag, New York, 1991. xvi+551 pp.

[26] A.R. Gover, Almost conformally Einstein manifolds and obstructions, in Differential geometry and its applications, 247-260, Matfyzpress, Prague, 2005. Electronic: arXiv:math/0412393

[27] A.R. Gover, Conformal Dirichlet-Neumann maps and Poincaré-Einstein Manifolds, SIGMA (Symmetry, Integrability and Geometry: Methods and Applications) 3100 (2007). arXiv:0710.2585

[28] A.R. Gover, Almost Einstein and Poincaré-Einstein manifolds in Riemannian signature, J. Geometry and Physics, 60 (2010), 182-204.

[29] A.R. Gover, in progress.

[30] A.R. Gover, and F. Leitner, A class of compact Poincare-Einstein manifolds: properties and construction, Communications in Contemporary Mathematics, to appear. arXiv:0808.2097

[31] A.R. Gover, and H. Macbeth, in progress.

[32] C.R. Graham, Volume and area renormalizations for conformally compact Einstein metrics. The Proceedings of the 19th Winter School "Geometry and Physics" (Srní, 1999), Rend. Circ. Mat. Palermo (2) Suppl. No. 63 (2000), 31-42.

[33] C.R. Graham, and J.M. Lee, Einstein metrics with prescribed conformal infinity on the ball, Adv. Math. 87 (1991), 186-225.

[34] C.R. Graham, and M. Zworski, Scattering matrix in conformal geometry, Invent. Math. 152 (2003), $89-118$.

[35] C.R. Graham, and E. Witten, Conformal anomaly of submanifold observables in AdS/CFT correspondence, Nuclear Phys. B 546 (1999), no. 1-2, 52-64.

[36] S.S. Gubser, I.R. Klebanov and A. M. Polyakov, Gauge theory correlators from non-critical string theory, Phys. Letters B 428 (1998), 105-114.

[37] I. Kolar, P.W. Michor, J. Slovák, Natural Operations in Differential Geometry, Springer 1993

[38] J.M. Lee, Fredholm operators and Einstein metrics on conformally compact manifolds, Mem. Amer. Math. Soc. 183 (2006), no. 864, vi+83 pp.

[39] F. Leitner, On transversally symmetric pseudo-Einstein and Fefferman-Einstein spaces, Math. Z. 256 (2007), 443-459.

[40] J. Lepowsky, A generalization of the Bernstein-Gelfand-Gelfand resolution, J. Algebra, 49 (1977), 496-511.

[41] J. Maldacena The large $N$ limit of superconformal field theories and supergravity, Adv. Theor. Math. Phys. 2 (1998), 231-252.

[42] R. Mazzeo and F. Pacard, Maskit combinations of Poincaré-Einstein metrics, Adv. Math. 204 (2006), 379-412.

[43] B. Orsted, Generalized gradients and Poisson transforms, Global analysis and harmonic analysis (Marseille-Luminy, 1999), 235-249, Sémin. Congr., 4, Soc. Math. France, Paris, 2000.

[44] R. Penrose and W. Rindler, Spinors and Space-time vol. 1, Cambridge University Press 1984.

[45] M. Randall, Almost projectively Ricci-flat manifolds, MSc thesis, University of Auckland, 2010.

[46] K. Skenderis, and S.N. Solodukin, Quantum effective action from the AdS/CFT correspondence, Phys. Lett. B472 (2000), 316-322, hep-th/9910023

[47] T.Y. Thomas, Announcement of a projective theory of affinely connected manifolds, Proc. Nat. Acad. Sci., 11 (1925), 588-589.

A. ̌. and M. H.: Faculty of Mathematics, University of Vienna, Nordbergstr. 15, 1090 Wien, Austria, A.R.G.:Department of Mathematics, The University of Auckland, Private Bag 92019, Auckland 1142, New Zealand; Mathematical Sciences Institute, Australian National University, ACT 0200, Australia

E-mail address: Andreas.Cap@univie.ac.at

E-mail address: r.gover@auckland.ac.nz

E-mail address: Matthias. Hammerl@univie.ac.at 